\title{
Eye detection using color cues and projection functions
}

\author{
R. Thilak kumar, S. Kumar Raja and A. G. Ramakrishnan' \\ Department of Electrical Engineering \\ Indian Institute of Science \\ Bangalore 560012 \\ E-mail: ramkiag@ee.iisc.ernet .in
}

\begin{abstract}
We propose a heuristic approach for detection of eyes in close-up images. The experimental images are stereotypical mug shot faces which can be expected in applications of face recognition systems, say in ATM vestibules. We prove the efficacy of our proposed melhod in delection of eyes, both in indoor and outdoorenvironments with considerablescale variations and an allowable rotation in the imageplane. We employ a hierarchical search spacereduction technique to localize possible eye areas in the image. Thedistinct human skin color andlow intensity areas of the eye bail are the primary cues used lo locate eye regions. Further on, eye validation is performed using the mean and varianceprojection functions.
\end{abstract}

Keywords : Normalized RGB. illumination normalization. connected component analysis, projection functions. eye detection.

\section{Introduction}

One of the most essential pre-requisites in building an automated system for face recognition is eye detection. Detecting the eyes eases the problem of locating other facial features such as nose and mouth required for recognition tasks. Eye detection :is invaluable in determining the orientation of the face and also the gaze direction. Eyes are important facial features due to their relatively constant interocular distance. The various schemes that have been proposed for eye detection can be broadly categorized into two approaches. The first assumes that the approximate eye regions are located or some constraints are imposed on the face image so that eye windows can be easily located. Eye detection is restricted to these windows. However. it is generally difficult to locate the eye windows in real world situations. In the second approach, a face detection algorithm $[1,2]$ is used to extract the approximate face region and

\footnotetext{
*This work was supported in pan by Samsung, SDS.Korea
}

the detection of eyes is carried out on the identified face area. However, several problems are associated with such approaches. The eye detection accuracy depends on the robustness of the face detection algorithm. Moreover, unless the orientation of the face is known, it is very difficult to extract the eye pair. The proposed scheme does not rely on any prior knowledge of possible eye windows nor is it preceded by a face detection algorithm. In our scheme, cues such as the distinct human skin color and low intensity of the eye are used to advantage.

The remainder of this paper is organized as follows. Section 2 briefly reviews related work. Section 3 provides a description of the proposed algorithm. Section 4 critically reviews the presented work. This is followed by conclusion.

\section{Earlier work on Eye Detection}

Ahuja et. al. [3] model human skin color as a Gaussian mixture and estimate model parameters using the Expectation Maximization algorithm. Yang et. al. [4] propose an adaptive bivariate Gaussian skin color model to locate human faces. Baluja et. al. [1] suggest a neural network based face detector with orientation normalization. Approaches such as this require exhaustive training sets. Pitas et. al. [2] use thresholding in HSV color space for skin color extraction. However, this technique is sensitive to illumination changes and race.

Huang et. al. [5] perform the task of eye detection using optimal wavelet packets for eye representation and radial basis functions for subsequent classification of facial areas into eye and non-eye regions. Rosenfeld et. al. [6] use filters based on Gabor wavelets to detect eyes in gray level images. Feng et. al. [7] employ multi cues for eye detection on gray images using variance projection function. However, the variance projection function on a eye window is not very consistent. Pitas et. al, [2] adopt a similar approach using the vertical and horizontal reliefs for the detection of the eye pair requiring pose normalization. 


\section{Proposed Method}

We achieve eye detection in three stages. In the first stage. possible eye areas are localized using a simple thresholding in HSV color space and normalized RGB color space. sequentially. It is followed by a connected component analysis to quantify spatially connected regions and further reduce the search space to determine the contending eye pair windows. Finally the mean and variance projection functions are employed in each eye pair window to validate the presence of the eye.

\subsection{Locating Possible Eye Areas}

The input face image is illumination normalized with a histogram stretching operation. It is essential to eliminate the noise in the image which manifests itself in the lowermost and uppermost ends of the histogram. To this end, we compute the cumulative sum of the histogram. from either end and discard pixels below $0.1 \%$ of the sum. The residual histogram is now stretched over the entire range. This operation is individually performed on each of the $R, G$ and $B$ channels. Figure 1 clarifies this operation. where the threshold is set to $1 \%$ for the purpose of clarity.

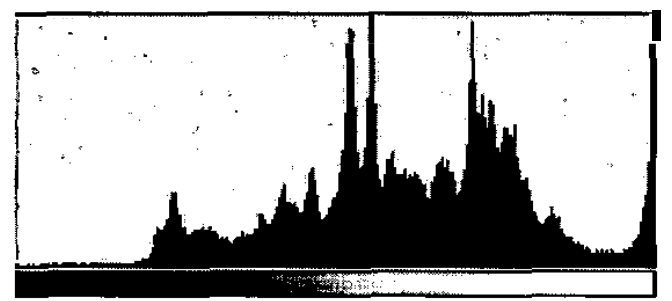

(a)

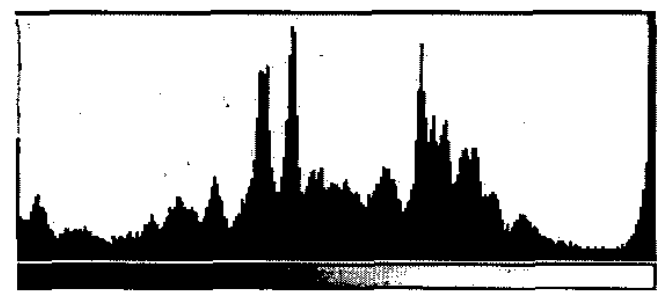

(b)

\section{Figure 1. (a) Histogram of the original image; (b) $1 \%$ threshold histogram stretching}

The possible eye regions are obtained in three stages. In the first stage. the illumination normalized image is transformed into the HSV space and low intensity areas detected by thresholding in $\mathrm{V}$. In our experiments, a $\mathrm{V}$ value of below 90 in a scale of 255 corresponds to the low intensity regions. It is seen that for dark faces, the skin colored pixels lie within this threshold. To eliminate these skin pixels, our second stage uses thresholding of the original image in the normalized RGB space which has proven to be effective. The thresholds used are described below:

$$
96.0<\text { norm, }<120.0
$$

where $75.0<$ norm $m_{g}<86.0$

$$
\begin{aligned}
& \text { norm }_{r}=(r /(r+\mathbf{g}+b)) * 255.0 \text { and } \\
& \text { norm }_{g}=(g /(r+\mathbf{g}+b)) * 255.0
\end{aligned}
$$

The low intensity regions in the entire image are identified by ANDing the results of the first two stages. In the third stage. a patch of the image area under each of the extracted possible eye regions. obtained by ANDing operation is analyzed for skin color. This is performed on the original image in the normalized RGB space with a wide threshold to accommodate various races. We assume that the skin color is not degraded due to any camera effects.

The thresholds used for skin analysis are

$$
\begin{aligned}
& 90.0<\text { norm }_{r}<145.0 \\
& 60.0<\text { norm }_{g}<100.0
\end{aligned}
$$

The thresholds were chosen after a lot of experimentation on wide rariety of images containing face of different races. Thus, we reduce the possible eye candidates further as shown in Fig. 5.

\subsection{Connected Component Analysis}

In real world scenes, several objects in the background appear as low-intensity areas in the image and sometimes with a skin-like region below. Also. in our thresholding scheme, the human hair is a possible eye candidate. To be rid of these contenders, we use a simple aspect ratio and population test on each of the possible eye areas. To use these tests. it is necessary to quantify each of the located eye areas. A connected component analysis groups or clusters spatially connected regions in the image. Each of the clusters are put to an aspect ratio test and spurious clusters eliminated. In our experiments, we require the cluster aspect ratio to lie above 0.75 . This test is proven effective in discarding the elongated areas corresponding to the hair and other background objects. Prior to performing the connected component analysis. a gray-level dilation improves pixel connectivity.

We make use of the anthropometric knowledge that eyes occur as a pair within a specific distance depending on the image size. The angles of the linesjoining the centroid of one cluster to every other cluster are measured along with the length of the lines to discriminate between possible eyepairs. Only those pair of clusters that lie within an angle and distance threshold are chosen as possible eye-pairs. The angle threshold is \pm 20 degrees and distance threstiold is $1 / 6$ th the width of the image,justifiable if the face occupies a minimum of $25 \%$ of the entire frame. It is observed that the nostrils are rejected by employing this set of thresholds.

The results at this stage are shown in Fig. 6. 


\subsection{Eye Validation}

The possible eye candidates at this stage are the eyebrows. eyes, ends of the lip and hair (if the hair cluster is split). Using horizontal projections. each of these low intensity pairs are segregated into analysis windows as shown in Fig. 7. In each of these analysis windows, vertical projections are used to divide the window into two parts, each consisting of a low intensity area. In the case of the actual eye-window, in each part of the analysis window, there are two spatially disconnected low intensity clusters corresponding to the eye and the eyebrow. In most realistic situations, it is difficult to detect skin area below the eyebrow and hence the eyebrow cluster is eliminated. In each of the analysis windows, the angle of the linejoining the centroids of the two clusters is used to normalize the window in orientation. Each of such windows is subjected to an enhancement scheme so as to increase the contrast between the eyes and skin. The contrast enhancement uses illumination normalization with a threshold of $1 \%$ as explained above. This process is preceded by median filtering and gray scale erosion to reduce the effect of noise.

At this juncture, we employ the idea of reliefs or mean projection function [MPF] of Pitas et. al. and variance projection function [VPF] of Feng et. al. to detect the nose bridge and eyes. Typical normalized mean and variance projections in the vertical direction along with their difference for the eye pair window is shown in Fig. 2. We argue that. if the peak in the MPF. the valley in the VPF, and the peak in the difference plot between the flat areas, all of these three corresponding to the nose bridge, lie approximately in the same vertical position. then the analysis window is an eye-window.

\section{Discussion}

The proposed method is successful in detecting eyes of varying scale, within a certain allowable image plane orientation. The detection scheme is insensitive to variations in human skin color and illumination to an extent. However. harsh variations in illumination that drastically change the appearance of skin color affect the performance of the algorithm. The algorithm is computationally efficient as it relies only on low level visual cues and simple thresholding. The drawback of the proposed scheme is that eyes can be detected only in frontal close-up images. However, in a constrained domain of application. the algorithm can be employed with confidence.

\section{Conclusion}

We present a simple and heuristic approach to the problem of eye detection in static color images. The method is devoid of any intensive computations and relies primarily on cues such as the low-intensity of the eye ball and skin color which are scale and orientation invariant.
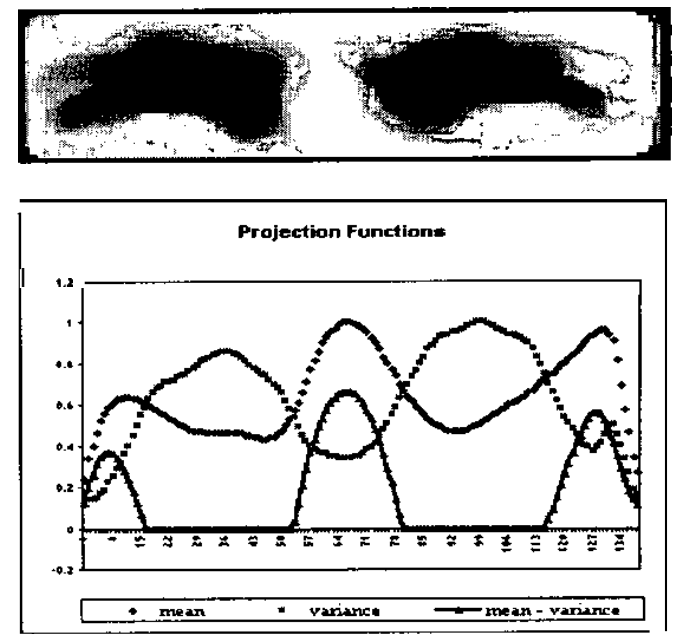

Figure 2. Enhanced eye pair window and its Projection functions

\section{References}

[1] H. Rowley. S. Baluja. and T. Kanade. "Neural Network-Based Face Detection," Proc.IEEE Conf. on Computer Vision and Pattern Recognition. San Francisco, CA, 1996.203-207.

[2] K. Sobattka and I. Pitas. "A Novel Method for Automatic Face Segmentation. Facial Feature Extraction and Tracking," Signal Processing:Image Communication, 12(3), 1998,263-281

[3] Ming-Hsuan Yang and Narendra Ahuja. "Detecting Human Faces in Color Images," Pmceedingsof IEEE Int 'l conf on Image Processing. Chicago, IL, 1998, 127-130.

(4) Jie Yang, Weier Lu and Alex Waibel. "Skin Color Modeling and Adaptation." Proceedings of ACCV'98 (tech report CMU-CS-97-146, CS dept. CMU. 1997).

[5] Jeffrey Huang and Harry Wechsler. "Eye Detection Using Optimal Wavelet Packets and Radial Basis Functions." International Journal of Pattern Recognition and Artificial Intelligence. Vol 13 No 7, 1999.

[6] S. A. Sirohey and Azriel Rosenfeld. "Eye detection in a face image using linear and nonlinear filters." Pattern Recognition.Vol 34. 2001, 1367-1391.

[7] Guo Can Feng and Pong C. Yuen. "Multi-cues eye detection on gray intensity image," Pattern Recognition. Vol 34, 2001, 1033-1046. 


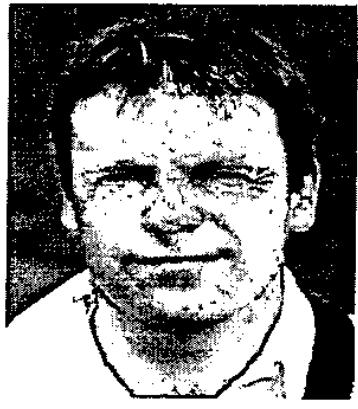

Figure 3. Original color image

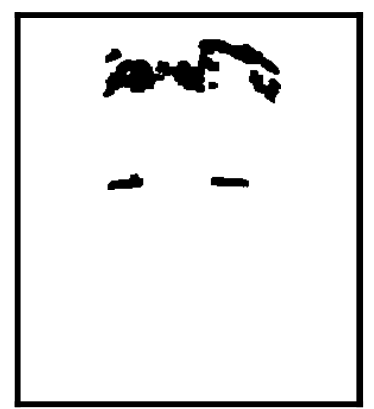

Figure 6. Possible Eye regions

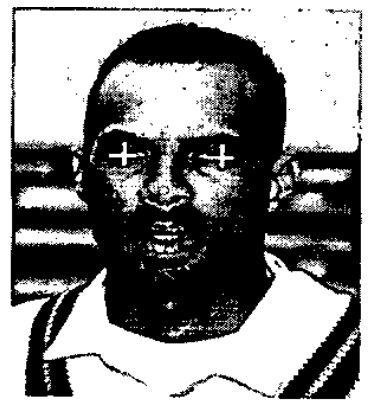

Figure 9. Result 2

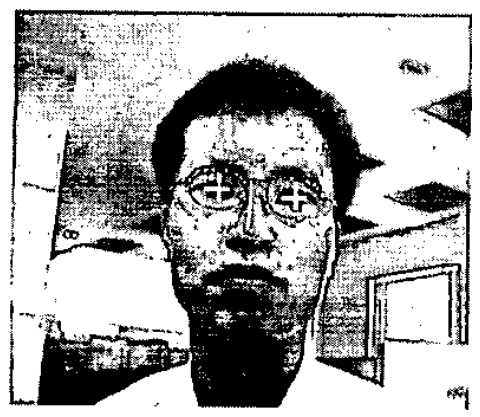

Figure 12. Result 5

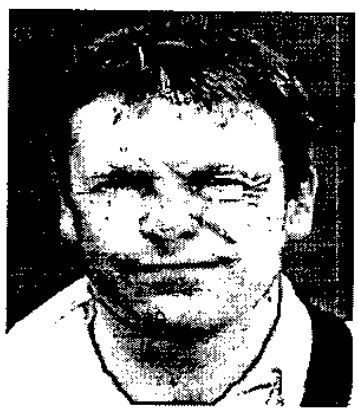

Figure 4. Illumination Normalized

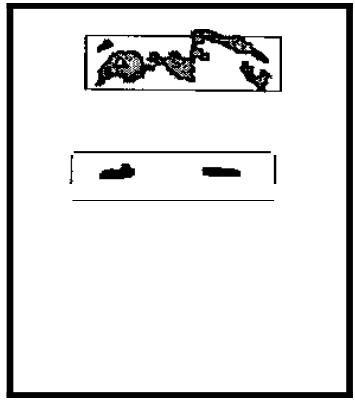

Figure 7. Analysis windows

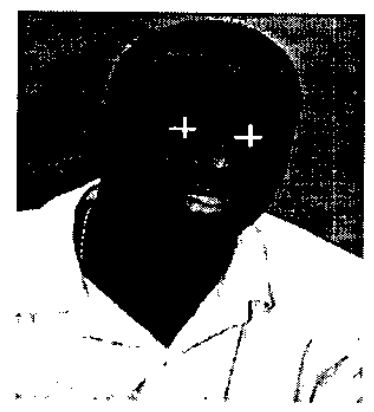

Figure 10. Result 3

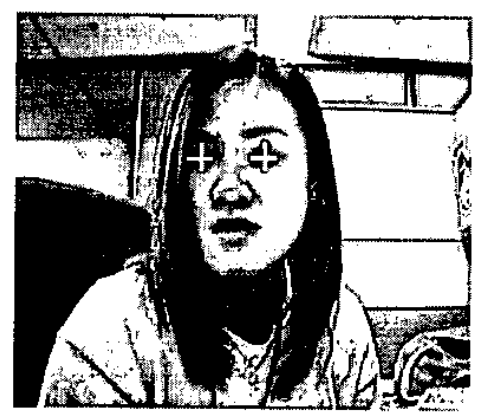

Figure 13. Result 6

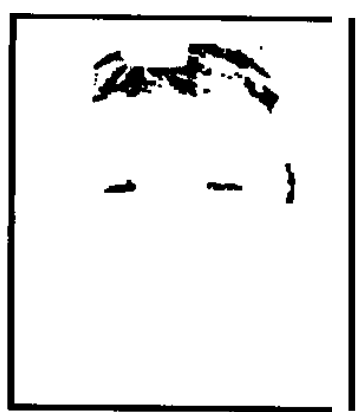

Figure 5. Retained Low intensity regions

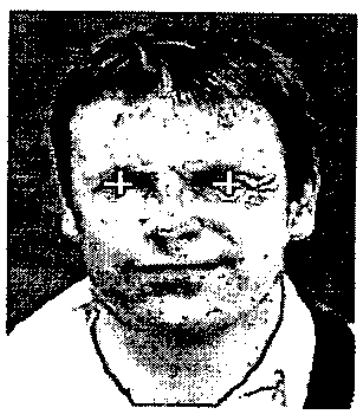

Figure 8. Detected Eye

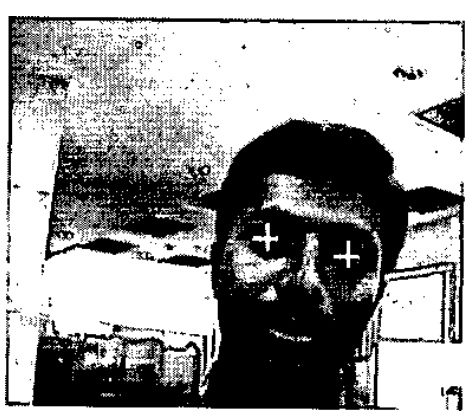

Figure 11. Result 4

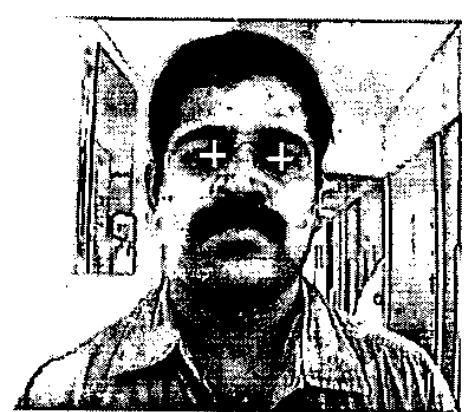

Figure 14. Result 7 